\section{Dor nos dentes e gengivas e fatores associados em adolescentes brasileiros: análise do inquérito nacional de saúde bucal SB-Brasil 2002-2003}

\author{
Dental and gingival pain and associated factors \\ among Brazilian adolescents: an analysis of the \\ Brazilian Oral Health Survey 2002-2003
}

\author{
1 Program de Pós-graduação \\ em Saúde Pública, \\ Universidade Federal \\ de Santa Catarina, \\ Florianópolis, Brasil. \\ 2 Faculdade de Odontologia, \\ Universidade de São Paulo, \\ São Paulo, Brasil. \\ Correspondência \\ K. G. Peres \\ Programa de Pós-graduação \\ em Saúde Pública, \\ Universidade Federal de \\ Santa Catarina. \\ Campus Universitário \\ Trindade, Florianópolis, SC \\ 88010-970, Brasil. \\ karengp@ccs.ufsc.br
}

\begin{abstract}
The aim of this study was to estimate the prevalence of dental and gingival pain and associated factors among Brazilian adolescents (15-19 years of age). Data from 16,126 adolescents who participated in the Brazilian Oral Health Survey SB-Brazil 2002-2003 were used. The outcome measured was dental and gingival pain in the last six months. Independent variables were per capita income, schooling, school enrollment, gender, skin color, age, area of residence, time since last dental appointment, type of dental service, DMFT index and its components, dental calculus, and Dental Aesthetic Index. Simple and multiple Poisson regression analyses were performed. Prevalence of dental and gingival pain was 35.6\% (95\%CI: 34.8-36.4). Increased prevalence of pain was associated with: female gender, low income, non-students, students enrolled in public schools, and grade-for-age lag. In addition, adolescents with high levels of dental caries and dental calculus also reported higher prevalence of dental pain. Dental and gingival pain can be considered a relevant public health problem, suggesting the need for preventive measures.
\end{abstract}

Toothache; Gingiva; Oral Health; Adolescent

\author{
Carolina Marques Borges 1 \\ Andreia Morales Cascaes ${ }^{1}$ \\ Tatiana Konrad Fischer 1 \\ Antonio Fernando Boing 2 \\ Marco Aurélio Peres 1 \\ Karen Glazer Peres 1
}

\section{Introdução}

A dor é uma experiência vivenciada por quase todas as pessoas. É por meio dela que a maioria das afecções se manifesta podendo ser expressa de diversos modos e em diferentes populações 1 . Fatores cognitivos, expectativas e crenças, fatores sociais, psicológicos e culturais podem afetar sua percepção 2,3 .

O comitê de taxonomia da International Associaton for the Study of Pain (IASP) ${ }^{4}$ conceitua a dor como uma experiência sensorial e emocional desagradável, decorrente ou descrita em termos de lesões teciduais.

A dor orofacial consiste em dor localizada abaixo da linha metaorbital, acima do pescoço e em posição anterior ao ouvido, sendo conceituada como uma condição dolorosa associada a tecidos duros e moles da cabeça, face, pescoço e todas as estruturas intra-orais 5 .

Entre as dores orofaciais, a dor de dente é a mais freqüente. Ela pode ser caracterizada como a dor que emana dos dentes e de suas estruturas de suporte, sendo resultado da cárie dentária, doença periodontal ou traumatismo, caracterizando-se como aguda, recorrente ou crônica ${ }^{6}$. A dor de dente tem uma substancial importância em Saúde Pública, pois dependendo de sua intensidade, pode causar impacto na vida diária dos indivíduos acometidos e na sociedade 2,7 . Entre esses efeitos, destacam-se os custos econômicos decorrentes, sejam eles diretos relativos 
aos serviços de saúde, ou indiretos, referentes à ausência no trabalho e diminuição da produtividade, bem como a ausência na escola, no caso de estudantes ${ }^{3,8}$.

Diversos fatores têm sido associados à dor de dente, dentre eles fatores sócio-econômicos, demográficos, fatores psicológicos, étnicos, culturais e padrões de acesso a serviços odontológicos e utilização deles. De acordo com a literatura, indivíduos com piores condições sócio-econômicas apresentam piores condições de vida e, possivelmente, apresentam maior número de agravos à saúde bucal, dentre eles, a dor de dente 9 . Em relação à cor da pele, as diferenças podem ser atribuídas às desigualdades sociais, observando-se que, geralmente, pretos e pardos tendem a apresentar as piores condições de vida e de saúde do que indivíduos brancos 2,10,11,12,13. Além disso, a dor de dente é um forte preditor da utilização dos serviços de saúde ${ }^{14}$.

O presente estudo objetivou investigar a prevalência de dor nos dentes e gengivas relatada nos últimos seis meses por adolescentes brasileiros e fatores a ela associados.

\section{Métodos}

Trata-se de estudo transversal exploratório realizado com base em dados secundários oriundos do levantamento epidemiológico nacional em saúde bucal - SB Brasil 2002-2003. O referido levantamento, realizado pelo Ministério da Saúde, investigou diferentes condições de saúde bucal de 108.921 brasileiros de diferentes grupos etários (18-36 meses de vida, 5, 12, 15-19, 35-44 e 6574 anos de idade), residentes em 250 municípios das zonas urbanas e rurais de todas as cinco macrorregiões do país. Indivíduos com idade igual ou maior que 15 anos foram examinados e entrevistados nos domicílios e investigados sobre suas condições sócio-econômicas, utilização dos serviços odontológicos e autopercepção da saúde bucal. As condições e os agravos à saúde bucal estudados foram a cárie dentária, as condições periodontais, o edentulismo, o uso e a necessidade de prótese, as anormalidades dentofaciais e a fluorose, baseados nos critérios estabelecidos pela Organização Mundial da Saúde (OMS) em 199715.

A amostragem foi probabilística por conglomerados. Em cada macrorregião do país, os municípios foram sorteados conforme sua inserção em cinco estratos definidos pelo tamanho de população (menos de 5.000 habitantes, 5.001$10.000,10.001-50.000,50.001-100$ mil e mais de 100.000), totalizando 250 municípios. Todas as capitais dos estados foram incluídas previamente ao sorteio e não participaram dele. Após o sorteio dos municípios, sortearam-se os setores censitários e as residências. Maiores informações sobre o processo de amostragem do SB-Brasil 20022003 constam no seu relatório final 15 .

A taxa de resposta para a faixa etária de 15-19 anos foi de $84,5 \%$, totalizando 16.833 adolescentes. Para o presente estudo, foram excluídos os indígenas e os de descendência asiática (amarelos), além dos indivíduos sem informação referente à cor da pele, devido ao pequeno número na amostra, portanto, a análise foi restrita a 16.126 adolescentes.

\section{Definição das variáveis}

A variável dependente investigada foi a "dor nos dentes e gengivas nos últimos seis meses”. A pergunta original do questionário utilizado no SBBrasil 2002-2003 foi: "O quanto de dor seus dentes e gengivas causaram nos últimos seis meses?”, com as seguintes opções de resposta: nenhuma dor, pouca, média e muita dor. Para o presente estudo, o desfecho foi dicotomizado em não (ausente) e sim (presente). As variáveis independentes testadas foram: condições sócio-econômicas (renda per capita, escolaridade, condição de estudo); localização geográfica da residência (urbana, rural); condições demográficas (sexo, idade, cor da pele); utilização de serviços odontológicos (tipo de serviço odontológico utilizado, tempo da última consulta) e; agravos bucais (cárie, perda dentária, cálculo dentário, índice de estética dental). A renda per capita (em Reais - R\$) foi calculada dividindo-se a renda familiar pelo número de habitantes do domicílio e categorizada segundo os quartis: $\leq \mathrm{R} \$ 40,00 ; \mathrm{R} \$ 41,00-80,00 ; \mathrm{R} \$$ 81,00-150,00 e; > R \$ 150,00. A condição de estudo foi categorizada em estudar em escola privada, pública e não estudar. A escolaridade foi calculada de acordo com os anos de estudo completos, a saber: $\leq 4,5-8,9-11$ e $\geq 12$. As variáveis sexo (masculino, feminino) e idade (15, 16, 17, 18 e 19 anos) foram mantidas como no banco original. A cor da pele foi recategorizada em branca, parda e preta. O tipo de serviço odontológico utilizado foi dicotomizado em público e privado. O tempo decorrente desde a última consulta odontológica foi mantido como originalmente encontrado no banco: nunca foi ao dentista, foi há menos de 1 ano, 1-2 anos, e há mais de 2 anos. $\mathrm{O}$ índice de dentes permanentes cariados, perdidos e obturados (CPO-D) foi categorizado segundo a sua distribuição em quartis: $\leq 2$, 3-5, 6-9 e $\geq 10$. O componente $\mathrm{C}$ do índice CPO-D foi dividido em tercis: $0,1-3, \geq 4$ e o componente $\mathrm{P}$ foi dicotomizado em $0 \mathrm{e} \geq 1$. As condições periodontais foram aferidas por intermédio do escore máximo do Ín- 
dice Periodontal Comunitário (CPI), que avalia a condição periodontal segundo higidez, presença de sangramento gengival, cálculo dentário e bolsas periodontais. Para o presente estudo, a variável cálculo dentário foi obtida pelo índice CPI e, posteriormente, dicotomizada em presença e ausência de cálculo. Por se tratar de adolescentes, o cálculo dentário foi considerado a pior condição, ou seja, a mais prevalente $(33,4 \%)$ e não a bolsa periodontal, uma vez que a presença das mesmas foi muito baixa $(1,3 \%)$ na faixa etária dos 15 aos 19 anos de idade. Finalmente, a variável Índice de Estética Dental (DAI) foi obtida como originalmente encontrada no banco de dados, classificada conforme as categorias preconizadas pela OMS 15 em: $\leq 25$, 26-30, 31-35 e $\geq 36$.

\section{Análise dos dados}

A primeira etapa da análise consistiu na realização das análises bivariadas das proporções da dor de dente e gengivas para cada variável independente, apresentando as razões de prevalência com os respectivos intervalos de 95\% de confiança (IC95\%).

A seguir, realizou-se a regressão de Poisson e foram estimadas as razões de prevalências brutas e ajustadas assim como seus respectivos IC95\% e teste de Fisher, por ser mais preciso e conservador. A prevalência do desfecho excedeu $20 \%$, justificando a utilização da regressão de Poisson alternativamente ao uso da regressão logística 16 . Apenas as variáveis que apresentaram valor de $\mathrm{p}<0,25$ nas análises brutas foram incluídas nas análises múltiplas 17 .

A modelagem estatística seguiu o modelo teórico hierárquico de determinação da dor de dente e gengivas (Figura 1). Inicialmente, as variáveis do bloco 1 (condições sócio-econômicas, localização demográfica da residência e fatores demográficos) foram ajustadas entre si. Em seguida, as variáveis do bloco 2 (utilização dos serviços odontológicos e agravos à saúde bucal) foram ajustadas entre si e pelas variáveis do bloco 1. Todas as análises foram realizadas pelo programa Stata 9.0 (Stata Corp., College Station, Estados Unidos) tendo sido ponderadas pelo efeito do delineamento amostral pelo comando svy destinado à análise de dados oriundos de amostras complexas. A adequação do modelo foi testada, indicando um bom ajuste ( $\mathrm{p}=1,00$ - goodenessof-fit-test).

\section{Questões éticas}

O projeto Condições de Saúde Bucal da População Brasileira - SB-Brasil 2002-2003 foi aprovado pelo Conselho Nacional de Ética em Pesquisas com Seres Humanos (CONEP), sob o parecer no. $581 / 2000$, em 21 de julho de 2000. Foi obtido o

Figura 1

Modelo teórico de determinação hierárquica da dor nos dentes e gengivas.

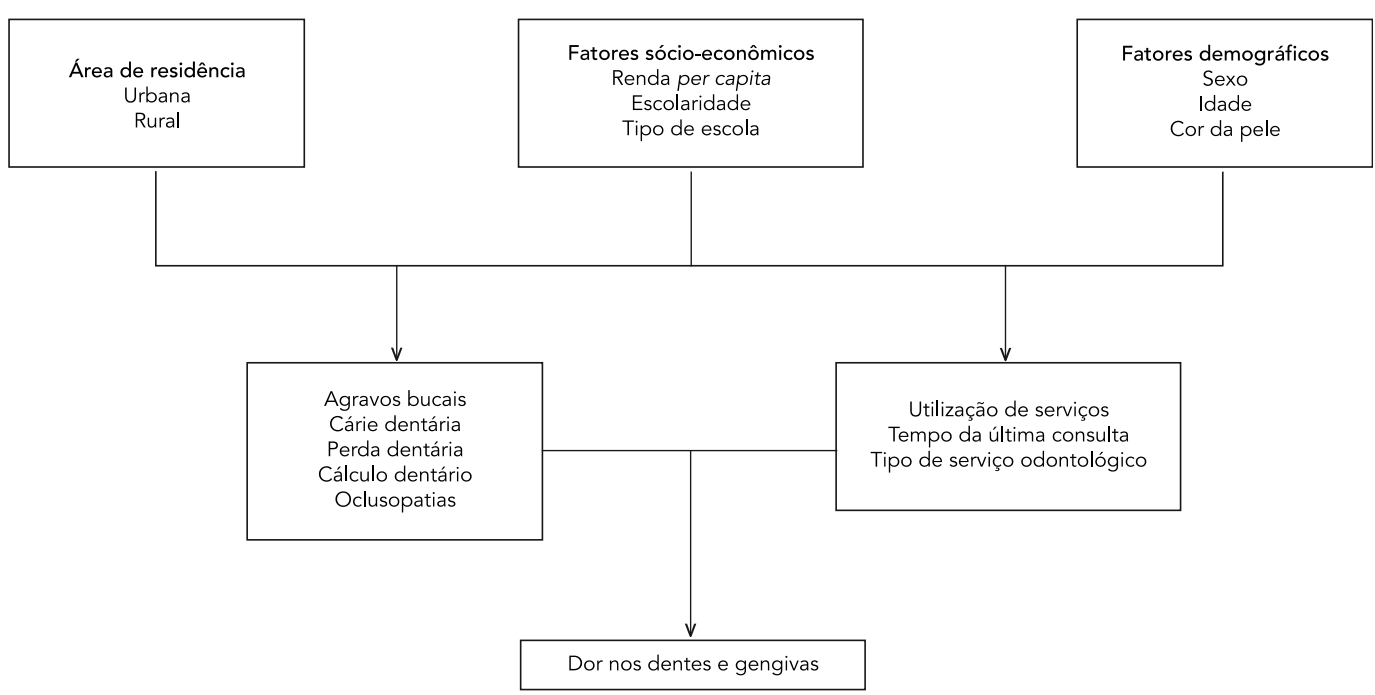


termo de consentimento de todos os indivíduos participantes do estudo.

\section{Resultados}

A prevalência de dor nos dentes e gengivas foi igual a 35,6 (IC95\%: 34,8-36,4). A Tabela 1 apresenta a distribuição na amostra e a prevalência de dor nos dentes e gengivas de acordo com as categorias das variáveis independentes. Adolescentes não estudantes $(40,2 \%)$, com menor escolaridade $(41,8 \%)$ e com menor renda per capita relataram mais dor nos dentes e gengivas que os demais. Verificou-se que a dor nos dentes e gengivas acometeu mais as meninas (37,8\%) que os meninos (32,6\%). A maior prevalência do desfecho foi verificada entre os indivíduos par- dos quando comparados aos pretos e brancos. Indivíduos com índice CPO-D $\geq 10$ apresentaram prevalência do desfecho duas vezes (46\%) maior que aqueles com CPO-D $\leq 2$ (23\%). A dor nos dentes e gengivas foi mais prevalente entre aqueles que apresentaram os piores agravos bucais (quatro ou mais dentes cariados, pelo menos um dente perdido por cárie e presença de cálculo dentário). Na análise bivariada, todas as variáveis foram fortemente associadas à dor $(\mathrm{p}<0,001)$, exceto a variável local de residência $(\mathrm{p}=0,923)$ como pode ser visto na Tabela 1.

A Tabela 2 apresenta as razões de prevalências brutas para cada variável independente e os modelos da análise múltipla. Após o ajuste entre as variáveis do bloco 1 , a cor da pele perdeu a significância estatística $(\mathrm{p}=0,074)$. Indivíduos com menor renda per capita, não estudantes, com

Distribuição da amostra e prevalência de dor nos dentes e gengivas segundo as variáveis do estudo e respectivos intervalos de $95 \%$ de confiança (IC95\%) em adolescentes brasileiros de 15 a 19 anos de idade $(\mathrm{N}=16.126)$.

\begin{tabular}{|c|c|c|c|c|c|}
\hline \multirow[t]{2}{*}{ Variáveis } & \multicolumn{2}{|c|}{ Distribuição na amostra } & \multicolumn{2}{|c|}{ Dor nos dentes e gengivas } & \multirow[t]{2}{*}{$p$} \\
\hline & n & $\%$ & Prevalência & IC95\% & \\
\hline $\operatorname{Dor}(n=15.969)$ & 5.948 & 35,6 & 35,6 & $34,9-36,4$ & \\
\hline Renda per capita (Reais) $[n=15.810]$ & & & & & $<0,001$ \\
\hline$>150,00$ & 3.942 & 24,9 & 31,8 & $30,4-33,2$ & \\
\hline $81,00-150,00$ & 3.767 & 23,9 & 35,1 & $33,5-36,7$ & \\
\hline $41,00-80,00$ & 4.096 & 26,0 & 38,3 & $36,8-39,8$ & \\
\hline$\leq 40,00$ & 4.005 & 25,2 & 36,8 & $35,0-38,5$ & \\
\hline Escolaridade (anos) $[\mathrm{n}=15.851]$ & & & & & $<0,001$ \\
\hline$\geq 12$ & 1.152 & 7,4 & 31,2 & $28,6-34,0$ & \\
\hline $9-11$ & 6.059 & 38,3 & 33,5 & $32,5-34,6$ & \\
\hline $5-8$ & 6.800 & 42,7 & 37,0 & $35,8-38,2$ & \\
\hline$\leq 4$ & 1.840 & 11,6 & 41,8 & $40,0-44,1$ & \\
\hline Local de residência [ $n=16.109]$ & & & & & 0,923 \\
\hline Urbana & 13.937 & 86,5 & 35,6 & $34,8-36,5$ & \\
\hline Rural & 2.172 & 13,5 & 36,2 & $33,8-37,7$ & \\
\hline Condição de estudo [ $n=15.834]$ & & & & & $<0,001$ \\
\hline Privada & 742 & 4,7 & 26,2 & $23,3-29,5$ & \\
\hline Pública & 10.945 & 69,1 & 34,8 & $33,9-35,7$ & \\
\hline Não estuda & 4.147 & 26,2 & 40,2 & $38,5-41,9$ & \\
\hline Sexo $[n=16.126]$ & & & & & $<0,001$ \\
\hline Masculino & 6.736 & 41,7 & 32,6 & $31,5-33,7$ & \\
\hline Feminino & 9.390 & 58,3 & 37,8 & $36,8-38,8$ & \\
\hline Idade (anos) $[\mathrm{n}=16.126]$ & & & & & $<0,001$ \\
\hline 15 & 4.606 & 28,5 & 33,5 & $32,2-34,9$ & \\
\hline 16 & 3.174 & 19,7 & 34,5 & $32,8-36,4$ & \\
\hline 17 & 2.814 & 17,6 & 37,1 & $35,2-39,1$ & \\
\hline 18 & 2.594 & 16,0 & 36,6 & $34,9-38,2$ & \\
\hline 19 & 2.938 & 18,2 & 37,7 & $36,1-39,5$ & \\
\hline
\end{tabular}

(continua) 


\begin{tabular}{|c|c|c|c|c|c|}
\hline \multirow[t]{2}{*}{ Variáveis } & \multicolumn{2}{|c|}{ Distribuição na amostra } & \multicolumn{2}{|c|}{ Dor nos dentes e gengivas } & \multirow[t]{2}{*}{$\mathrm{p}$} \\
\hline & $\mathrm{n}$ & $\%$ & Prevalência & IC95\% & \\
\hline Cor da pele $[n=16.126]$ & & & & & $<0,001$ \\
\hline Branca & 7.071 & 43,8 & 33,6 & $32,5-34,7$ & \\
\hline Parda & 7.369 & 45,7 & 37,4 & $36,3-38,6$ & \\
\hline Preta & 1.686 & 10,5 & 36,2 & $33,8-38,5$ & \\
\hline Tempo desde a última consulta (anos) $[n=15.890]$ & & & & & $<0,001$ \\
\hline Menos de 1 & 7.720 & 48,5 & 37,1 & $36,0-38,1$ & \\
\hline $1-2$ & 3.710 & 23,3 & 34,9 & $33,4-36,5$ & \\
\hline 3 ou mais & 2.338 & 14,7 & 38,3 & $36,5-40,2$ & \\
\hline Nunca foi atendido & 2.122 & 13,5 & 29,1 & $27,1-31,1$ & \\
\hline Tipo de serviço odontológico $[n=13.410]$ & & & & & $<0,001$ \\
\hline Privado & 4.525 & 33,9 & 33,2 & $31,9-34,5$ & \\
\hline Sistema Único de Saúde (SUS) & 8.885 & 66,1 & 38,4 & $37,3-39,4$ & \\
\hline Índice CPO-D [n = 16.126] & & & & & $<0,001$ \\
\hline$\leq 2$ & 4.167 & 25,7 & 23,0 & $21,7-24,3$ & \\
\hline $3-5$ & 4.071 & 25,2 & 36,4 & $34,9-37,9$ & \\
\hline $6-9$ & 4.325 & 26,8 & 38,6 & $37,2-40,0$ & \\
\hline$\geq 10$ & 3.563 & 22,3 & 46,0 & $44,0-47,7$ & \\
\hline Componente $\mathrm{C}$ do $\mathrm{CPO}-\mathrm{D}[\mathrm{n}=16.126]$ & & & & & $<0,001$ \\
\hline 0 & 5.506 & 34,2 & 22,7 & $21,6-23,8$ & \\
\hline $1-3$ & 5.638 & 34,9 & 37,1 & $35,9-38,4$ & \\
\hline$\geq 4$ & 4.982 & 30,9 & 48,2 & $46,8-49,6$ & \\
\hline Componente P do CPO-D $[n=16.126]$ & & & & & $<0,001$ \\
\hline 0 & 10.241 & 63,4 & 31,2 & $30,3-32,1$ & \\
\hline$\geq 1$ & 5.885 & 36,6 & 43,3 & $42,0-44,6$ & \\
\hline Cálculo dentário [n = 12.846] & & & & & $<0,001$ \\
\hline Não & 7.441 & 58,0 & 30,5 & $29,5-31,5$ & \\
\hline Sim & 5.405 & 42,0 & 40,9 & $39,5-42,3$ & \\
\hline Índice DAI [ $\mathrm{n}=16.126]$ & & & & & $<0,001$ \\
\hline$\leq 25$ & 7.525 & 46,8 & 34,1 & $33,1-35,2$ & \\
\hline $26-30$ & 3.313 & 20,4 & 35,4 & $33,7-37,1$ & \\
\hline $31-35$ & 2.260 & 18,0 & 35,9 & $33,8-38,0$ & \\
\hline$\geq 36$ & 3.028 & 18,8 & 39,4 & $37,6-41,1$ & \\
\hline
\end{tabular}

Notas: valor de $p=$ teste exato de Fisher; todos os valores foram ajustados pelo efeito do delineamento.

baixa escolaridade, do sexo feminino e mais velhos apresentaram maiores prevalências de dor nos dentes e gengivas.

Após o ajuste das variáveis do bloco 2 entre si e pelas variáveis do bloco 1 , verificou-se que ter ido ao dentista há três anos ou mais, alto ataque de cárie (índice $\mathrm{CPO}-\mathrm{D} \geq 10$, componente $\mathrm{C}$ do CPO-D $\geq 4$ e componente $\mathrm{P} \geq 1$ ) e presença de cálculo dentário permaneceram associadas às maiores prevalência do desfecho. A variável índice DAI associou-se à dor no limite da significância estatística ( $\mathrm{p}=0,051$ ), e a prevalência de dor nos dentes e gengivas foi maior nos indivíduos com oclusopatias mais graves (DAI $\geq 36$ ) do que nos demais.

\section{Discussão}

Existem poucos estudos internacionais e nacionais de base populacional que investigaram a prevalência da dor de dente em adolescentes. A prevalência da dor de dentes e gengivas em adolescentes brasileiros nos últimos seis meses foi de $35,6 \%$. A alta prevalência (37\%) de dor nos dentes também foi encontrada em 2001 em adolescentes da Finlândia usando-se recordatório de 24 meses 13 , porém esse resultado sugere uma maior prevalência no Brasil, visto que o recordatório do presente estudo foi de seis meses. No Município de Florianópolis, Santa Catarina, Brasil, 21,2\% dos adolescentes do sexo masculino, aos 18 anos de idade, relataram dor de dente nos últimos 12 meses, desde a entrevista, no ano de 200418. 
Tabela 2

Associação entre dor nos dentes e gengivas e variáveis sócio-econômicas, demográficas, utilização dos serviços odontológicos e condições de saúde bucal em adolescentes brasileiros de 15 a 19 anos de idade. Razões de prevalência (RP), intervalos de 95\% de confiança (IC95\%) e valores de p (regressão Poisson).

\begin{tabular}{|c|c|c|c|c|c|c|c|c|c|}
\hline \multirow[t]{2}{*}{ Variáveis } & \multicolumn{2}{|c|}{ Análise bruta } & \multirow[t]{2}{*}{$p$} & \multicolumn{2}{|c|}{ Modelo 1} & \multirow[t]{2}{*}{$\mathrm{p}$} & \multicolumn{2}{|c|}{ Modelo 2} & \multirow[t]{2}{*}{$p$} \\
\hline & RP & IC95\% & & RP & IC95\% & & RP & IC95\% & \\
\hline \multicolumn{10}{|l|}{ Bloco 1} \\
\hline Renda per capita (Reais) & & & $<0,001$ & & & 0,001 & & & \\
\hline$>150,00$ & 1,0 & & & 1,0 & & & & & \\
\hline $81,00-150,00$ & 1,1 & $1,0-1,2$ & & 1,0 & $1,0-1,1$ & & & & \\
\hline $41,00-80,00$ & 1,2 & $1,1-1,3$ & & 1,1 & $1,1-1,2$ & & & & \\
\hline$\leq 40,00$ & 1,2 & $1,1-1,2$ & & 1,1 & $1,0-1,2$ & & & & \\
\hline Escolaridade (anos) & & & $<0,001$ & & & $<0,001$ & & & \\
\hline$\geq 12$ & 1,0 & & & 1,0 & & & & & \\
\hline $9-11$ & 1,1 & $1,0-1,2$ & & 1,1 & $1,0-1,2$ & & & & \\
\hline $5-8$ & 1,2 & $1,1-1,3$ & & 1,2 & $1,0-1,3$ & & & & \\
\hline$\leq 4$ & 1,3 & $1,2-1,5$ & & 1,3 & $1,1-1,4$ & & & & \\
\hline Condição de estudo & & & $<0,001$ & & & $<0,001$ & & & \\
\hline Privada & 1,0 & & & 1,0 & & & & & \\
\hline Pública & 1,3 & $1,2-1,5$ & & 1,2 & $1,1-1,4$ & & & & \\
\hline Não estuda & 1,5 & $1,3-1,7$ & & 1,3 & $1,2-1,5$ & & & & \\
\hline Sexo & & & $<0,001$ & & & $<0,001$ & & & \\
\hline Masculino & 1,0 & & & 1,0 & & & & & \\
\hline Feminino & 1,2 & $1,1-1,2$ & & 1,2 & $1,1-1,2$ & & & & \\
\hline Idade (anos) & & & $<0,001$ & & & $<0,001$ & & & \\
\hline 15 & 1,0 & & & 1,0 & & & & & \\
\hline 16 & 1,0 & $1,0-1,1$ & & 1,0 & $1,0-1,1$ & & & & \\
\hline 17 & 1,1 & $1,0-1,2$ & & 1,1 & $1,1-1,2$ & & & & \\
\hline 18 & 1,1 & $1,0-1,2$ & & 1,1 & $1,0-1,2$ & & & & \\
\hline 19 & 1,1 & $1,0-1,2$ & & 1,1 & $1,0-1,2$ & & & & \\
\hline Cor da pele & & & $<0,001$ & & & 0,074 & & & \\
\hline Branca & 1,0 & & & 1,0 & & & & & \\
\hline Parda & 1,1 & $1,0-1,2$ & & 1,1 & $1,0-1,1$ & & & & \\
\hline Preta & 1,1 & $1,0-1,2$ & & 1,0 & $1,0-1,1$ & & & & \\
\hline \multicolumn{10}{|l|}{ Bloco 2} \\
\hline \multicolumn{10}{|l|}{ Tempo desde a última } \\
\hline consulta (anos) & & & $<0,001$ & & & & & & 0,037 \\
\hline$<1$ & 1,0 & & & & & & 1,0 & & \\
\hline $1-2$ & 1,0 & $0,9-1,0$ & & & & & 0,9 & $0,8-1,0$ & \\
\hline 3 ou mais & 1,0 & $1,0-1,1$ & & & & & 1,0 & $0,9-1,1$ & \\
\hline Nunca foi atendido & 0,8 & $0,7-0,8$ & & & & & 0,8 & $0,7-0,8$ & \\
\hline Tipo de serviço odontológico & & & $<0,001$ & & & & & & 0,909 \\
\hline Privado & 1,0 & & & & & & 1,0 & & \\
\hline SUS & 1,2 & $1,1-1,2$ & & & & & 1,0 & $1,0-1,1$ & \\
\hline Índice CPO-D & & & $<0,001$ & & & & & & 0,006 \\
\hline$\leq 2$ & 1,0 & & & & & & 1,0 & & \\
\hline $3-5$ & 1,6 & $1,5-1,7$ & & & & & 1,0 & $1,1-1,3$ & \\
\hline $6-9$ & 1,7 & $1,6-1,8$ & & & & & 1,2 & $1,1-1,3$ & \\
\hline$\geq 10$ & 2,0 & $1,6-2,1$ & & & & & 1,3 & $1,2-1,4$ & \\
\hline Componente C do CPO-D & & & $<0,001$ & & & & & & $<0,001$ \\
\hline 0 & 1,0 & & & & & & 1,0 & & \\
\hline $1-3$ & 1,6 & $1,5-1,7$ & & & & & 1,5 & $1,4-1,6$ & \\
\hline$\geq 4$ & 2,1 & $2,0-2,2$ & & & & & 1,7 & $1,6-1,8$ & \\
\hline
\end{tabular}

(continua) 


\begin{tabular}{|c|c|c|c|c|c|c|c|c|c|}
\hline \multirow[t]{2}{*}{ Variáveis } & \multicolumn{2}{|c|}{ Análise bruta } & \multirow[t]{2}{*}{$\mathrm{p}$} & \multicolumn{2}{|c|}{ Modelo 1} & \multirow[t]{2}{*}{$p$} & \multicolumn{2}{|c|}{ Modelo 2} & \multirow[t]{2}{*}{$p$} \\
\hline & $\mathrm{RP}$ & $1 \mathrm{C95 \%}$ & & $\mathrm{RP}$ & IC95\% & & RP & $\mathrm{IC} 95 \%$ & \\
\hline Componente P do CPO-D & & & $<0,001$ & & & & & & $<0,001$ \\
\hline 0 & 1,0 & & & & & & 1,0 & & \\
\hline$\geq 1$ & 1,4 & $1,3-1,4$ & & & & & 1,1 & $1,0-1,1$ & \\
\hline Cálculo dentário & & & $<0,001$ & & & & & & $<0,001$ \\
\hline Não & 1,0 & & & & & & 1,0 & & \\
\hline Sim & 1,3 & $1,3-1,4$ & & & & & 1,1 & $1,1-1,2$ & \\
\hline Índice DAI & & & $<0,001$ & & & & & & 0,051 \\
\hline$\leq 25$ & 1,0 & & & & & & 1,0 & & \\
\hline $26-30$ & 1,0 & $1,0-1,1$ & & & & & 1,0 & $1,0-1,1$ & \\
\hline $31-35$ & 1,1 & $1,0-1,1$ & & & & & 1,0 & $1,0-1,1$ & \\
\hline$\geq 36$ & 1,2 & $1,1-1,2$ & & & & & 1,1 & $1,0-1,1$ & \\
\hline
\end{tabular}

Notas: análise bruta = valores brutos; valor de $\mathrm{p}=$ teste de Wald; todas as análises foram ajustadas pelo efeito do delineamento.

Modelo 1: variáveis do bloco 1 ajustadas entre si;

Modelo 2: variáveis do bloco 2 ajustadas entre si e pelas variáveis do bloco 1.

Apesar das elevadas taxas de prevalência de dor de dente verificadas nos estudos citados, há limitações para a comparação entre os mesmos em razão das diferentes metodologias empregadas como, por exemplo, diferenças nas faixas etárias estudadas, tipos de instrumentos utilizados, período e tempo relativo à medida dos desfechos. Quanto maior o tempo investigado, maior a possibilidade de esquecimento ou subestimação da dor. A utilização de um recordatório com tempo inferior como, por exemplo, o de um mês, poderia facilitar a lembrança do entrevistado, estimando de maneira mais precisa a prevalência do desfecho. Por outro lado, outros tipos de dores como as orofaciais, por exemplo, possibilitaram a superestimação da dor de dente relatada, pois na impossibilidade de identificação precisa da origem da dor, os demais tipos de dores poderiam ser confundidos como dor de dente.

A associação entre condição sócio-econômica e dor nos dentes e gengivas foi verificada no presente estudo, corroborando com estudos anteriores 2,18,19. Entretanto, tal associação não é consenso na literatura científica ${ }^{20}$. Indivíduos pertencentes aos grupos sócio-econômicos de menor renda e menor escolaridade têm piores condições de vida, menos acesso e menor utilização dos serviços de saúde e, conseqüentemente, possuem as piores condições bucais, inclusive a dor de dente. Sabe-se que o componente psicossocial também pode influenciar a dor, uma vez que a privação e o estresse causados por piores condições de vida poderão potencializá-la 2 .

No que se refere aos fatores demográficos, a prevalência de dor nos dentes e gengivas foi maior entre as meninas comparadas aos meni- nos. Resultados semelhantes podem ser observados em outros estudos com populações adultas 19,21 , todavia, por se tratar de faixas etárias distintas, comparações não podem ser realizadas diretamente com o presente estudo. Tal fato pode ser explicado pela maior prevalência de cárie e perdas dentárias entre as mulheres do que nos homens, justificando o maior relato de dor de dente entre as mesmas. Geralmente, o maior relato de dor de dente sentida entre as mulheres pode relacionar-se aos níveis mais altos de estresse psicológico, ansiedade e depressão 22 . Em adição, a superação da dor pelas mulheres é mais difícil do que nos homens 23 , entretanto os estudos ainda são inconclusivos quanto à associação entre gênero e dor de dente.

A variável cor da pele perdeu a significância estatística após o ajuste pelas variáveis demográficas e sócio-econômicas indicando, neste estudo, que cor da pele pode ter sido confundida pelas variáveis sociais e econômicas.

No presente estudo, nunca ter ido ao dentista foi considerado, aparentemente, um fator de proteção, porém, provavelmente, trata-se de causalidade reversa, um dos problemas inerentes aos estudos transversais. Sabe-se que a dor de dente é um fator de predição para a utilização dos serviços odontológicos, já que a sensação dolorosa estimula a visita ao dentista 24 . Além disso, indivíduos que utilizam serviços odontológicos com maior freqüência tendem a amenizar ou mesmo prevenir os quadros de dor e, assim, relatarão menos o desfecho do que aqueles que utilizam os serviços com menor freqüência 25 .

O tipo de serviço odontológico utilizado na última consulta não se associou à dor nos dentes 
e gengivas quando se observa os modelos ajustados, indicando a importância do controle por possíveis variáveis de confusão. Os resultados sugerem que ter utilizado o Sistema Único de Saúde (SUS) na última consulta odontológica, não implica maior prevalência do desfecho.

A cárie pode ser considerada uma das principais causas da dor de origem dentária. A associação positiva entre cárie e dor de dente foi encontrada no presente estudo concordando com outras pesquisas 18,26. Dentre as condições clínicas analisadas, a prevalência da dor nos dentes foi maior entre os indivíduos com mais de quatro dentes cariados e com, pelo menos, uma perda dentária por causa de cárie. O componente P do índice CPO-D reflete a extensão e a gravidade da cárie 14 , justificando a sua associação com o desfecho.

Outra condição clínica associada positivamente à dor nos dentes e gengivas foi a presença de cálculo dentário aferido pelo índice CPI. Geralmente, a presença de cálculo relaciona-se à higienização precária dos dentes. Indivíduos com higiene bucal deficiente poderão apresentar maior número de dentes cariados e, conseqüentemente, relatarão mais dor de dente. Contudo, mesmo após o ajuste por cárie, a presença do cálculo manteve-se associada ao desfecho, sugerindo a existência de dor nas gengivas.

$\mathrm{O}$ sangramento gengival poderia influenciar o desfecho, mas não foi considerado na análise por conta das limitações inerentes à classificação do índice CPI, que considera como escore máximo a pior condição periodontal de cada sextante. Dessa maneira, ao considerar o cálculo dentário como pior condição periodontal, subestima-se a prevalência de sangramento gengival. Por outro lado, a presença de bolsas periodontais maiores ou iguais a $4 \mathrm{~mm}$ apresentou-se rara $(1,34 \%)$ nesta população, conferindo ao cálculo dentário pior condição periodontal sem a subestimação de sua prevalência.

A presença de oclusopatias, medida pelo índice DAI, manteve-se no limite da significância estatística, e a chance de sentir dor nos dentes e gengivas foi maior nos indivíduos com oclusopatias mais severas (DAI $\geq 36$ ) do que nos demais. Sabe-se que a presença de algum tipo de oclusopatia não provoca dor nos dentes e gengivas diretamente, porém podem ser considerados como um fator local coadjuvante. O mau posicionamento dentário tem sido associado à cárie dentária 27 assim como o apinhamento dentário, que favorece o aparecimento de inflamação gengival com possível manifestação dolorosa 28 .

O presente estudo apresentou algumas limitações como o delineamento transversal que não permitiu verificar a relação de causalidade entre as variáveis investigadas e o desfecho. Outra limitação inerente a um estudo que utiliza dados secundários refere-se à impossibilidade de controle da qualidade da coleta dos dados. O trauma dentário pode influenciar a dor nos dentes, entretanto o inquérito SB-Brasil não pesquisou tal agravo, o que também pode ser considerado uma limitação. Do mesmo modo, o desfecho deste estudo, na verdade, corresponde a dois desfechos "dor nos dentes" e "dor nas gengivas", no entanto o mais adequado seria obter os dois desfechos separadamente.

É importante ressaltar os pontos positivos desta pesquisa. O tamanho da amostra, sua representatividade populacional e o tipo de análise estatística realizada apresentam-se como pontos fortes do presente estudo. O SB-Brasil 2002-2003 foi um levantamento epidemiológico financiado pelo Ministério da Saúde e envolveu milhares de profissionais durante a sua execução. Utilizar os dados oriundos do SB-Brasil 2002-2003 é um compromisso junto à população em prol da busca de respostas mais conclusivas para o SUS, além de incentivar a melhoria dos levantamentos futuros 15 .

A dor de dente apresenta-se como um problema de saúde pública porque além da alta prevalência pode gerar impacto negativo na qualidade de vida dos indivíduos e na sociedade em razão do alto custo dos tratamentos curativos, falta ao trabalho ou à escola e o uso de medicamentos 29,30. Considerando-se a grande extensão territorial do Brasil e suas profundas desigualdades sociais, os impactos negativos na vida dos indivíduos causados pela dor de dente deverão reforçar a necessidade de ações prioritárias na política atual de saúde bucal de modo a garantir o princípio da eqüidade, ampliando o acesso da atenção e do cuidado àqueles com piores condições sócio-econômicas e de saúde. 


\section{Resumo}

Estimou-se a prevalência de dor nos dentes e gengivas e fatores associados em adolescentes brasileiros de 15 a 19 anos de idade. Foram utilizadas informações de 16.126 adolescentes participantes do levantamento epidemiológico nacional de saúde bucal - SB Brasil 2002-2003. O desfecho foi o relato de dor nos dentes e gengivas nos últimos seis meses. As variáveis exploratórias foram: renda per capita, escolaridade, condição de estudo, sexo, cor da pele, idade, localização geográfica da residência, tipo de serviço odontológico utilizado pela última vez, tempo decorrido da última consulta odontológica, índice CPO-D e seus componentes, cálculo dentário e o índice de estética dental. Foram realizadas análises brutas e múltiplas utilizando a regressão de Poisson. A prevalência da dor de dentes e gengivas foi de 35,6\% (IC95\%: 34,8-36,4). A prevalência de dor foi maior nas meninas, naqueles pertencentes a famílias com baixa renda per capita, nos não estudantes e estudantes de escola pública e naqueles com baixa escolaridade para a idade. Indivíduos que apresentaram altos níveis de cárie e cálculo dentário também apresentaram maiores prevalências do desfecho. A dor nos dentes e gengivas em adolescentes pode ser considerada um problema relevante em saúde pública sugerindo a necessidade de ações preventivas e de promoção da saúde.

Odontalgia; Gengiva; Saúde Bucal; Adolescente

\section{Referências}

1. Teixeira MJ. Epidemiologia clínica da dor. Rev Med (São Paulo) 1999; 78:36-54.

2. Slade GD. Epidemiology of dental pain and dental caries among children and adolescents. Community Dent Health 2001; 18:219-27.

3. Teixeira MJ, Braum Filho JL, Márquez JO, Yeng LT, organizadores. Dor: contexto interdisciplinar. $\mathrm{Cu}-$ ritiba: Editora Maio; 2003.

4. International Association for the Study of Pain. Part III: pain terms, a currente list with definitions and notes on usage. http://www. iasp-pain.org/AM/Template.cfm?Section= Pain_Definitions\&Template $=/$ CM $/$ HTMLDisplay. cfm\&ContentID =1728 (acessado em 04/ Abr/2007).

5. Okeson JP. Neuroanatomia funcional e fisiologia do sistema mastigatório. In: Okeson JP, organizador. Tratamento das desordens temporomandibulares e oclusão. São Paulo: Editora Artes Médicas; 2000. p. $42-9$

\section{Colaboradores}

C. M. Borges, A. M. Cascaes e T. K. Fischer participaram da escolha do tema, da construção do banco de dados, realização da análise estatística, organização e edição final do artigo. A. F. Boing, M. A. Peres e K. G. Peres participaram de todas as etapas acima citadas e colaboraram na discussão e revisão crítica da versão final do manuscrito.
6. Mumford L. Orofacial pain: aetiology, diagnosis and treatment. Londres: Churchill Livingstone; 1982.

7. Macfarlane TV, Blinkhorn AS, Davies RM, Kincey J, Worthington HV. Oro-facial pain in the community: prevalence and associated impact. Community Dent Oral Epidemiol 2002; 30:52-60.

8. Gift HC, Reisine ST, Larach DC. The social impact of dental problems and visits. Am J Public Health 1992; 82:1663-8.

9. Nomura LH, Bastos JLD, Peres MA. Dental pain prevalence and association with dental caries and socioeconomic status in schoolchildren, Southern Brazil, 2002. Braz Oral Res 2004; 18:134-40.

10. Sternbach RA. Survey of pain in the United States: the Nuprin Pain Report. Clin J Pain 1986; 2:49-53.

11. Locker D, Grushka M. Prevalence of oral and facial pain and discomfort: preliminary results of mail survey. Community Dent Oral Epidemiol 1987; 15:169-72. 
12. Lipton JA, Ship JA, Larach-Robinson D. Estimated prevalence and distribution of reported orofacial pain in the United States. J Am Dent Assoc 1993; 124:115-21.

13. Honkala E, Honkala S, Rimpelä A, Rimpelä M. The trend and risks factors of perceived toothache among finnish adolescents from 1977 to 1997 . J Dent Res 2001; 80:1823-7.

14. Lacerda JT, Simionato EM, Peres KG, Peres MA, Traebert J, Marcenes W. Dor de origem dental como motivo de consulta odontológica em uma população adulta. Rev Saúde Pública 2004; 38:453-8.

15. Coordenação de Saúde Bucal, Departamento de Atenção Básica, Secretaria de Atenção à Saúde, Ministério da Saúde. Projeto SB Brasil 2003. Condições de saúde bucal da população brasileira: 2002 2003. Resultados principais. Brasília: Ministério da Saúde; 2004.

16. Barros AJ, Hirakata VN. Alternatives for logistic regression in cross-sectional studies: an empirical comparison of models that directly estimate the prevalence ratio. BMC Med Res Methodol 2003; $3: 21$.

17. Hosmer DW, Lemeshow S. Applied logistic regression. New York: John Wiley and Sons; 1989.

18. Bastos JLD, Nomura LH, Peres MA. Dental pain, socioeconomic status, and dental caries in young male adults from southern Brazil. Cad Saúde Pública 2005; 21:1416-23.

19. Bastos JL, Gigante DP, Peres KG. Toothache prevalence and associated factors: a population based study in southern Brazil. Oral Dis 2008; 14:320-6.

20. Vigild M, Petersen PE, Hadi R. Oral health behaviour of 12-year-old children in Kuwait. Int J Paediatr Dent 1999; 9:23-9.

21. Unell L, Soderfeldt B, Halling A, Birkhed D. Explanatory models for clinically determined and symptom-reported caries indicators in an adult population. Acta Odontol Scand 1999; 57:132-8.
22. Dao TT, LeResche L. Gender differences in pain. J Orofac Pain 2000; 14:169-84.

23. Liddeir A, Locker D. Gender and age differences in attitudes of dental pain and dental control. Community Dent Oral Epidemiol 1997; 25:314-8.

24. Alexandre GC, Nadanovsky P, Lopes CS, Faerstein E. Prevalência e fatores associados à ocorrência da dor de dente que impediu a realização de tarefas habituais em uma população de funcionários públicos no Rio de Janeiro, Brasil. Cad Saúde Pública 2006; 22:1073-8.

25. Bolin AK. Children's dental health in Europe. An epidemiological investigation of 5- and 12-yearold children from eight EU countries. Swed Dent J Suppl 1997; 122:1-88.

26. Evans DJ, Rugg GA, Tabari ED, Butler T. The effect of fluoridation and social class on caries experience in 5-year-old Newcastle children in 1994 compared with results over the previous 18 years. Community Dent Health 1996; 13:5-10.

27. Antunes JL, Peres MA, Jahn GM, Levy BB. The use of dental care facilities and oral health: A multilevel approach of schoolchildren in the Brazilian context. Oral Health Prev Dent 2006; 4:287-94.

28. Gábris K, Marton S, Madlena M. Prevalence of malocclusion in Hungarian adolescents. Eur J Orthod 2006; 28:467-70.

29. Jokovic A, Locker D, Stephens M, Kenny D, Tompson B, Guyatti G. Validity and reliability of a questionnaire for measuring child oral-health-related quality of life. J Dent Res 2002; 81:459-63.

30. Feitosa S, Colares V, Pinkham J. The pschosocial effects of severe caries in 4-year-old children in Recife, Pernambuco, Brazil. Cad Saúde Publica 2006; 21:1550-6.

Recebido em 27/Set/2007

Versão final reapresentada em 19/Dez/2007

Aprovado em 14/Jan/2008 